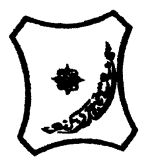

Bayero Journal of Pure and Applied Sciences, 12(1): 63 - 70

ISSN $2006-6996$

\title{
STUDIES ON Streptococcus pneumoniae ASSOCIATED WITH PULMONARY INFECTION AMONG HIV CLIENTS ATTENDING AMINU KANO TEACHING HOSPITAL (AKTH)
}

\author{
Mahmud, ${ }^{1}$ A. H., Dutsinma, U. A. ${ }^{1}$ and Idris, A. M. ${ }^{2}$ \\ ${ }^{1}$ Department of Microbiology, Faculty of Life Sciences, Bayero University, Kano Nigeria \\ ${ }^{2}$ Department of Medical Microbiology and Parastology, Faculty of Clinical Science, Bayero University, \\ Kano Nigeria \\ Tel: +2348103927920: abdulrahmanhabib01gmail.com
}

\begin{abstract}
Respiratory infection is highly prevalent in HIV-infected patients and S. pneumoniae was a leading cause. The aim of this study was to isolate and identify Streptococcus pneumoniae associated with pulmonary infection among HIV Clients Attending Aminu Kano Teaching Hospital (AKTH). A total of 300 samples comprising sample from 180 HIV seropositive patients and 120 HIV seronegative patients as a (control) were enrolled in this study.) For the 180 HIV seropositive patients, 105(58.3\%) were females, and 75(41.7\%) were males. Among the HIV negative subjects 64(53.3\%) were males and $56(46.7 \%)$ were females. The incident of Streptococcus pneumoniae isolates associated with pneumonia in HIV/AIDS seropositive patients in relation to age group in this study showed the higher prevalence of 15(51.72\%), among the age groups of 30-39, had the highest of prevalence of 15(51.72\%), which occur more common in HIV infected patients while for HIV seronegative patients age group 10-19 had the highest incidence of $7(29.17 \%)$.Result showed no significant difference in the prevalence of $S$ .pneumoniae among the HIV seropositive and seronegative patients $(P>0.05)$ .Antimicrobial susceptibility showed that Ciprofloxacin 25(86.2\%), was found to be most active invitro against the isolates followed by Oxacillin 21(72.4\%), Augmentin19 (65.5), Chloramphenicol 15(51.7\%). Tetracycline, 14(48.3\%), Clindamycin 14(48.3) and Erythromycin 10(34.5\%). Result showed no significant difference in the levels of antibiotic susceptibilities and resistance observed $(\mathrm{p}>0.05)$. The $\mathrm{CD}_{4}$ cells counts for the HIV seropositve patients were 161(89.4\%) 350 cells/mI and 19(10.6\%) $<350 \mathrm{cells} / \mathrm{m}$ recorded as normal and low respectively. There was significant difference $(p<0.05)$ for the CD4 cells counts of the study population with the prevalence of Streptococcus pneumoniae isolates among the HIV seropositive patients (P 0.000 <0.05). PCR amplification for Streptococcus pneumoniae penicillin resistance genes showed that Only 2(33.3\%) out of 6 resistant isolates possed the pneumococcal pbp2b resistance gene. According to the findings, the pbp2b gene can play a role of fundamentalimportance in the resistance of $S$. pneumoniae. Modify heterogeneity in antibiotic use, which several antibiotics are taken in a rotation against taking just 1 antibiotic such that isolates resistant to 1 antibiotic are killed when the subsequent antibiotic is taken.

Keywords: HIV/AIDS, Streptococcus Pneumoniae, Antibiotic Resistance, Minimal Inhibitory Concentration (MIC), Pbp2b CD4 cells count.
\end{abstract}

\section{INTRODUCTION}

Human immunodeficiency virus (HIV) infection and AIDS increase the risk of invasive pneumococcal disease (IPD) (Cohen et al., 2010). Underlying HIV infection is an important risk factor for pneumonia morbidity and mortality (Theodoratou et al., 2014). Streptococcus pneumoniae is the leading bacterial opportunistic infection in HIV positive Over two million People die each year worldwide and it is estimated that up to 1 million of these deaths are caused by the bacteria $S$. individuals (Thornhill et al., 2014).It is well known that patients with HIV have an increased risk of respiratory tract infections. Pneumonia carries a high mortality rate in immune compromised patients (Adeleye et al., 2008). Pneumonia is common, occurring in all age groups, and is a leading cause of death among the young, the old, and the chronically ill patients (Rano et al., 2008).

pneumoniae, and over $90 \%$ of these deaths take place in developing countries. The incidence of bacterial pneumonia in HIV-infected patients 
Special Conference Edition, November, 2019 ranges from 1.93 to $19.2 \%$ cases per 100 patients per year. The effect of HIV on the immune system is monitored by measuring the CD4 (T-helper) lymphocyte count in the blood. Depletion of CD4 cell count is a hallmark of disease progression in AIDS. CD4 cell count is essential to decide about the timing of initiation of antiretroviral therapy and for prophylaxis of opportunistic infections (Huang et al., 2010). The pandemic has lead to increased morbidity and mortality and created widespread resistance of bacteria to antibiotics among populations. Pneumonia continues to be an important problem in HIV infected patient (Ochei and Kolkhtar, 2008). The objective of the study is to determine the prevalence of Streptococcus pneumoniae associated with pulmonary infection among HIV/AIDS seropositive clients attending Aminu Kano Teaching Hospital (AKTH).

\section{MATERIALS AND METHODS}

Sampling Population and Ethical Clearance Ethical clearance was obtained from the Ethical committee of Aminu Kano Teaching Hospital (AKTH). HIV patients were recruited from Imam Wali Medical Center for virology (AKTH) Kano state Nigeria. Control were healthy employees with negative serology or no known risk factors for HIV infection but suspected to be Pneumonia patients.

\section{Sample Size}

Sample size was calculated to be 300 (180 from HIV/AIDS seropositive patients and 120 samples from HIV negative seronegative patients as a control) based on previously published studies by Ojo and Oluyege (2014) using Open Epi version 2.3 statistical software.

\section{Inclusion Criteria}

For the HIV seropositive patient (i) Consented to participate in the study (ii) Coughing

For the seronegative patients (i) Consented to participate in the study (ii) Coughing

\section{Culture and Processing of Sample}

Using a sterile microbiological loop a muco purulent part of the sputum sample was picked and inoculated onto blood agar and chocolate agar. The plates were incubated overnight at $37^{\circ} \mathrm{C}$. The colonies were identified using their growth patterns and colonial qmorphology on the agar plates. The organisms were identified by the appropriate biochemical tests such as Optochin test, Bile solubility test.

\section{Antibiotic Susceptibility Testing}

The following antibiotic disks were used:Tetracycline $(30 \mu \mathrm{g})$, Chloramphenicol $(30 \mu \mathrm{g})$,
Erythromycin $(15 \mu \mathrm{g}), \quad$ Ciprofloxacin $(5 \mu \mathrm{g})$, Clindamycin $(2 \mu \mathrm{g})$, Oxacillin $(1 \mu \mathrm{g})$ and Augmentin (30 $\mathrm{gg})$. Antibiotics susceptibility testing was performed according to standard procedures by CLSI (2010) (Disc agar diffusion method).

\section{Disk diffusion}

All isolates were tested for antibiotic susceptibility using the disk diffusion method according to CLSI standard with seven antimicrobial disks(Oxoid) Identified bacterial colonies were emulsified in sterile distilled water to conform to 0.5 McFarland turbidity standard, which was then diluted ten times to give a density of approximately 106 CFU per $\mathrm{ml}$ for inoculation on Mueller Hinton agar (OXOID, UK). Using a sterile cotton swab, the suspension was uniformly swabbed on Muller Hintoin agar (MH) (Pronadisa, Conda Laboratories, S.A).

\section{Polymerase Chain Reaction (PCR)}

Isolates of $S$. pneumoniae found to be resistant to oxacillin antibiotics were removed from the culture medium through scraping with a platinum spatula and placed into a tube with 50 $\mathrm{ml}$ of distilled water for extraction of bacterial DNA. Oligonucleotide initiators (primers) were used in the selection of initiators derived from the lytA gene of $S$. pneumoniae and from the genes encoding pbp2b of oxacillin antibiotics . Described as follows: $5 \mathrm{ml}$ of the buffer specific for Taq DNA polymerase, $2 \mathrm{mM}$ of $\mathrm{MgCl}_{2}, 1 \mathrm{U}$ of Taq DNA polymerase $5 \mathrm{U} / \mathrm{ml}$ (Gibco, Gaithersburg, MD, USA),200 mM of an equimolar mixture of nucleotide triphosphates and 50pmol of the forward primer (pbp2b-F ) CTTTGTCCCAGGTTCGGTTG and Reverse primer (pbp2b-R) CCCAAGCCATATTCGCCAAA of the two pairs of primers. For the preparation of the sample to be amplified positive controls, were used.Two $2 \mathrm{ml}$ of the DNA harvested from S. pneumoniae were mixed with $48 \mathrm{ml}$ of the master mixture. Regarding the amplification cycles, the samples prepared with the respective positive and negative controls were placed in the thermo cycler and submitted to 35 cycles, after an initial denaturation at $94^{\circ} \mathrm{C}$ for 3 minutes. Each cycle consisted of denaturation at $94^{\circ} \mathrm{C}$ for 30 seconds, annealing at $59^{\circ} \mathrm{C}$ for 30 seconds and extension at $72^{\circ} \mathrm{C}$ for 2 minutes. After the 35 amplification cycles. Agarose gel stained with $0.05(\%)$ ethidium bromide was prepared with wells and mounted in an electrophoretic tank. 
Special Conference Edition, November, 2019

Visualization of the bands was done using ultraviolet transilluminator (UVP, Upland USA) and pictures of the gel were taken using transmitted illumination camera fitted with a black and white Polaroid film (Sedigheh, et al., 2017).

\section{Primers used for Amplification of Resistance genes of Penicillin pbp2b. The pbp2b Gene}

was Successfully Amplified by PCR.

\begin{tabular}{lll}
\hline Gene & Primer Sequence $\left(5^{\prime} ! 3^{\prime}\right)$ & $\begin{array}{l}\text { Product, } \\
\text { Length, bp }\end{array}$ \\
\hline $\begin{array}{ll}\text { pbp2b-F } \\
\text { pbp2b-R }\end{array}$ & CTTTGTCCCAGGTTCGGTTG & 320 \\
\hline $\begin{array}{l}\text { Key: pbp2b-F = Forward primer } \\
\text { pbp2b-R }=\text { Reverse primer }\end{array}$ &
\end{tabular}

\section{Data Analysis}

The data generated in this study was analyzed using Statistical Package for Social Sciences(SPSS) (Version 25.0 IBM, Huz). Chisquare analysis ( $2 \times 2$ Statistical Analysis was used to determine the Prevalence of Streptococcus Pneumoniae isolates between HIV seropositive patients and seronegative patients used as a control for the Study. Chi-square analysis for antimicrobial resistance and susceptibility profile patterns of Streptococcus pneumoniae isolates between HIV/AIDS seropositive and HIV seronegative patients, while correlation was used for the CD4 cells counts of the study population with the prevalence of Streptococcus pneumoniae isolates in HIV seropositive patients.

\section{RESULTS}

\section{Gender Distribution of Subjects}

Three hundred (300) samples used for the study, made up of 180 HIV seropositive and 120 HIV seronegative patients as a control. Highest number of participants were obtained between the age groups $30-39$ years 75 $(41.7 \%), \quad 10-19$ years $7(29.17 \%), 40-59$ years $48(26.7 \%)$ and $25(20.8 \%)$ in both HIV seropositive and HIV seronegative respectively. Married participants from both groups have the highest number with 93(51.7\%), 74(61.7\%) respectively, while the least number were found in widow participants with $15(8.3 \%), 5(4.2 \%)$ respectively. $145(80.6 \%)$ were already on treatment for HIV seropositve patients. Also for cough Related infection, both HIV seropositive and HIV seronegative patients almost all patients were reported with a cough related infection as follows $179(99.4 \%) 1(0.6 \%) 120$ $(100.0 \%)$ respectively. Lastly the $\mathrm{CD}_{4}$ cells counts for the HIV seropositve patients were $161(89.4 \%) \quad>350$ cells $/ \mathrm{ml}$ and $19(10.6 \%)$ $<350$ cells $/ \mathrm{m}$ recorded as normal and low respectively (Table 1 ).

Among the HIV seropositive patients, (29) bacterial isolates of Streptococcus pneumoniae, were isolated. Highest prevalence were obtained in the age group $30-3915(51.72), 40-49$ 4(13.79), 50-59 4(13.79), 20-29 3(10.34), 60-69 $2(6.81), \quad 10-191(3.45)$ and 70-79 0(0.00) respectively . Among the HIV seronegative patients, (24) bacterial isolates of Streptococcus pneumoniae, were isolated were obtained in the age group. Highest prevalence were 10-19 7(29.17), 40-49 6(25.00), 20-29 4(16.67), 50-99 4(16.67), 60-69 2(8.33), 30-391(4.17) and 7079(0.00) respectively (Table 2 )

Percentage of antibiotics sensitivity profile for $S$. pneumoniae isolates for HIV seropositive patients. It shows that Ciprofloxacin 25(86.2\%), was most active invitro against the isolates followed by Oxacillin 21(72.4\%),Augmentin19 (65.5), Chloramphenicol 15(51.7\%), Tetracycline,14(48.3\%), Clindamycin14(48.3) and Erythromycin 10(34.5\%). Also the resistance profile for $S$. pneumoniae isolates for HIV seropositive patients. From the result obtained isolates showed a high rate of resistance of erythromycin $17(58.6 \%)$, followed by Chloramphenicol,12(41.4\%), Tetracycline9(31.0\%), Clindamycin 8(27.6), Augmentin7(24.1) and Oxacillin 6(20.7\%) while the least was Ciprofloxacin 1(3.5\%). Lastly the result for the intermediate antibiotics profile for S. pneumoniae isolates from HIV seropositive patients. From the result it shows that Clindamycin 7(24.1) Tetracycline 6(20.7\%), Ciprofloxacin3(10.3\%) Augmentin 3(10.3) was found out to be more effective than Chloramphenicol 2(6.9\%), Erythromycin $2(6.9 \%)$ and Oxacillin $2(6.9 \%)$ respectively (Table 3).

Percentage of antibiotics sensitivity profile for $S$. pneumoniae isolates for HIV seronegative patients. It showed that Oxacillin20(83.3\%), Augmentin 20(83.3\%), Ciprofloxacin18(75.0\%), Clindamycin 15(62.5), Erythromycin13(54.2\%) Tetracycline $11(45.8 \%)$ were highly sensitive while the least was Chloramphenicol I7(29.2\%) in HIV seronegative patients, respectively. Also the resistance profile for $S$. pneumoniae isolates from HIV seronegative patients. 
Special Conference Edition, November, 2019

It also showed that Chloramphenicol has a highly rate of resistance $10(41.7)$ followed by Tetracycline 8(33.3), Erythromycin 7(29.2) Clindamycin 7(29.2) Ciprofloxacin 4(16.7) while the least were Oxacillin 3(12.5) and Augmentin 3(12.5) respectively. Lastly the result for the intermediate antibiotics profile for $S$. pneumoniae isolates from HIV seronegative patients. From the result it showed that Tetracycline 5(20.8), Chloramphenicol, 7(29.2), Erythromycin,4(16.7) were more intermediate than Oxacillin, 3(12.5), Ciprofloxacin 2(8.3)
Clindamycin 2(8.3) and Augmentin 1(4.2) in HIV seronegative patients respectively. (Table 4)

The pbp2b gene was successfully amplified by PCR. Only two(2) (33.3\%) out of six(6) resistant isolates were observed penicillin resistance genes that detect the pneumococcal pbp2b genes. Primers were used in the selection of initiators derived from gene of $S$. pneumoniae and from the genes encoding pbp2b of penicillin-resistant gene of $S$. pneumoniae. The positive samples produced a visible band in the following sizes: pbp2b, 320 primers Figure I

Table 1 : Demographic information of subject enrolled among (HIV Seropositive and Seronegative Patients)

\begin{tabular}{|c|c|c|c|c|}
\hline Variables & $\begin{array}{l}\text { HIV Pos No (\%) } \\
\mathrm{N}=180\end{array}$ & & $\begin{array}{l}\text { HIV Neg No (\%) } \\
\text { (Control) } \quad \mathrm{N}=120\end{array}$ & \\
\hline \multicolumn{5}{|l|}{ Age Group (years) } \\
\hline $10-19$ & $1(0.6)$ & & $33(27.5)$ & \\
\hline $20-29$ & $14(7.8)$ & & $16(13.3)$ & \\
\hline $30-39$ & 75 (41.7) & & $12(10.0)$ & \\
\hline $40-49$ & $48(26.7)$ & & $25(20.8)$ & \\
\hline $50-59$ & $29(16.1)$ & & $17(14.4)$ & \\
\hline $60-69$ & $13(7.2)$ & & $15(12.5)$ & \\
\hline $70-79$ & $0(0.0)$ & & $2(1.7)$ & \\
\hline \multicolumn{5}{|l|}{ Sex } \\
\hline Male & 75 (41.7) & & $64(53.3)$ & \\
\hline \multicolumn{5}{|l|}{ Marital Status } \\
\hline Single & $72(40.0)$ & & $41(34.2)$ & \\
\hline Married & 93 (51.7) & & 74 (61.7) & \\
\hline Widow & $15(8.3)$ & & $5(4.2)$ & \\
\hline \multicolumn{5}{|l|}{ Cough Related infection } \\
\hline Yes & $179(99.4)$ & & $120(100.0)$ & \\
\hline & $1(0.6)$ & & $0(0.0)$ & \\
\hline \multicolumn{5}{|l|}{ On Treatment } \\
\hline Yes & $145(80.6)$ & & $120(100.0)$ & \\
\hline \multirow{2}{*}{\multicolumn{5}{|c|}{$\mathrm{CD}_{4}$ Count }} \\
\hline & & & & \\
\hline Normal & $161(89.4)$ & & Nil & \\
\hline Low & $19(10.6)$ & & Nil & \\
\hline \multicolumn{5}{|c|}{ Neg $=$ Negative $\%=$ Percentage } \\
\hline \multicolumn{5}{|c|}{$\begin{array}{l}\text { Table 2: Distribution of } S \text {, pneumoniae in relation to age groups between of HIV } \\
\text { seropositive and seronegative patients (control) }\end{array}$} \\
\hline Age groups (years) & Number & $(\%)(+V E)$ & Number & $(\%)(-V E)$ \\
\hline $10-19$ & 1 & 3.45 & 7 & 29.17 \\
\hline $20-29$ & 3 & 10.34 & 4 & 16.69 \\
\hline $30-39$ & 15 & 51.72 & 1 & 4.17 \\
\hline $40-49$ & 4 & 13.79 & 6 & 25.00 \\
\hline $50-59$ & 4 & 13.79 & 4 & 16.67 \\
\hline $60-69$ & 2 & 6.81 & 2 & 8.33 \\
\hline $70-79$ & 0 & 0.00 & 0 & 0.00 \\
\hline Total & 29 & 100 & 29 & 100 \\
\hline
\end{tabular}


Special Conference Edition, November, 2019

Table 3: Antimicrobial Susceptibility Patterns of Streptococcus pneumoniae Isolates from HIV/AIDS Seropositive Patients at AKTH

\begin{tabular}{llll}
\hline Antibiotics ( $\boldsymbol{\mu g})$ & Sensitive $\mathbf{n}(\%)$ & Intermediate $\mathbf{n}(\%)$ & Resistant $\mathbf{n}(\%)$ \\
\hline Tetracycline (30) & $14(48.3)$ & $6(20.7)$ & $9(31.0)$ \\
Cholrampinicol (30) & $15(51.7)$ & $2(6.9)$ & $12(41.4)$ \\
Erythromycin (15) & $10(34.5)$ & $2((6.9)$ & $17(58.6)$ \\
Ciprofloxacin (5) & $25(86.2)$ & $3(10.3)$ & $1(3.5)$ \\
Oxacillin (1) & $21(72.4)$ & $2(6.9)$ & $6(20.7)$ \\
Augmentin (30) & $19(65.5)$ & $3(10.3)$ & $7(24.1)$ \\
Clindamycin (2) & $14(48.3)$ & $7(24.1)$ & $8(27.6)$ \\
\hline
\end{tabular}

Table 4: Antimicrobial Susceptibility Patterns of Streptococcus Pneumoniae Isolates From HIV/AIDS Seronegative Patients At AKTH. (Control)

\begin{tabular}{llll}
\hline Antibiotics ( $\mu \mathbf{g})$ & Sensitive n (\%) & Intermediate n (\%) & Resistant n (\%) \\
& & & \\
\hline Tetracycline (30) & $11(45.8 \%)$ & $5(20.8)$ & $8(33.3)$ \\
Chlorampinicol(30) & $7(29.2 \%)$ & $7(29.2)$ & $10(41.7)$ \\
Erythromycin (15) & $13(54.2 \%)$ & $4(16.7)$ & $7(29.2)$ \\
Ciprofloxacin (5) & $18(75.0 \%)$ & $2(8.3)$ & $4(16.7)$ \\
Oxacillin (1) & $20(83.3 \%)$ & $3(12.5)$ & $1(4.2)$ \\
Augmentin (30) & $20(83.3)$ & $1(4.2)$ & $3(12.5)$ \\
Clindamycin (2) & $15(62.5)$ & $2(8.3)$ & $7(29.2)$ \\
\hline
\end{tabular}

Key: No=Number

$\%=$ Percentage

Amplification of Resistance Genes Pbp2b by PCR

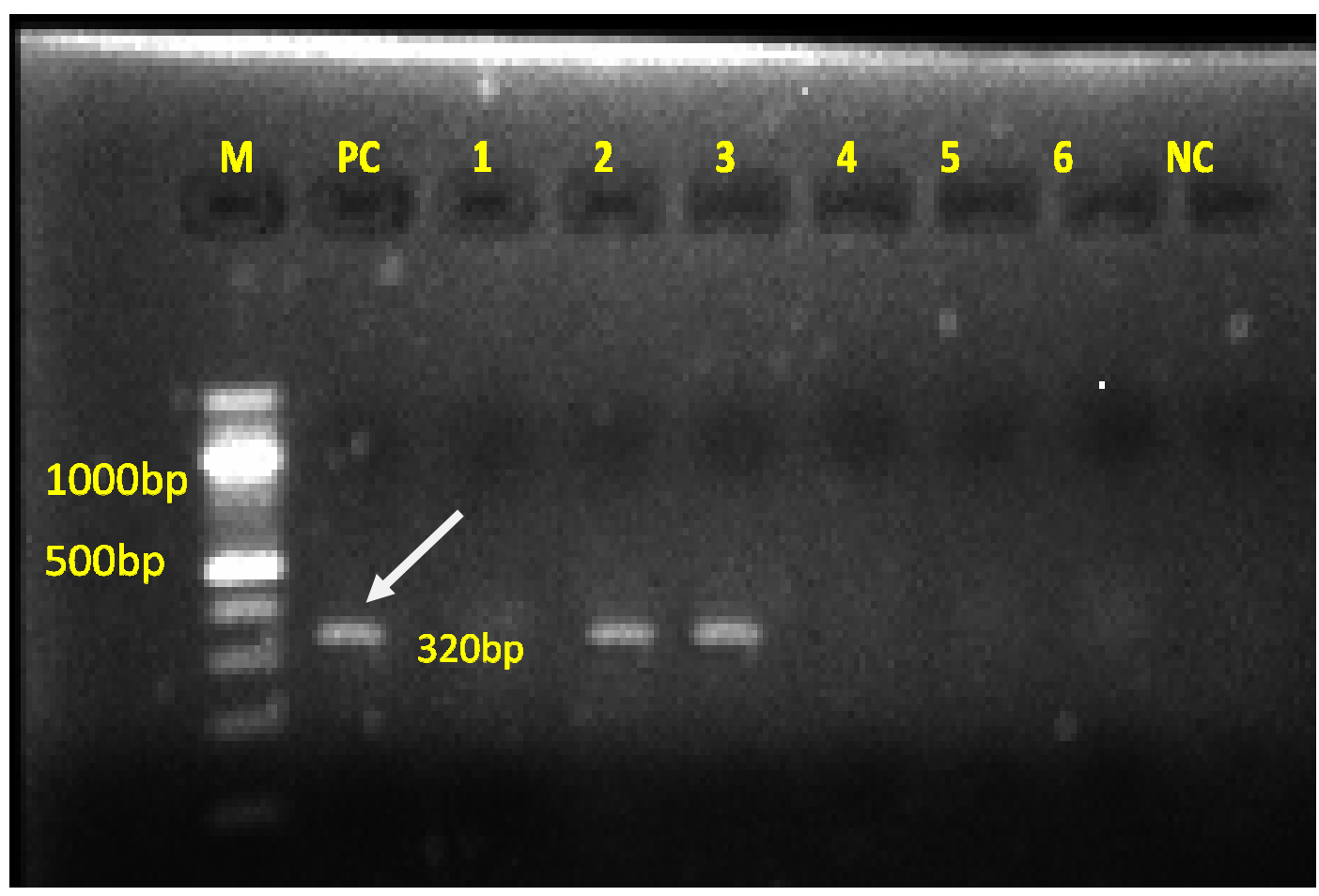

Plate III. Agarose Gel Electrophoresis for PCR - Amplified Fragments of the Pbp2b Genes Key

$M=$ Ladder 1000

$\mathrm{Bp} ; \mathrm{PC}=$ Positive Control

$\mathrm{NC}=$ Negative Control 


\section{Special Conference Edition, November, 2019 DISCUSSION}

Pneumonia is a common illness in all parts of the world. Streptococcus pneumoniae remains a leading cause of morbidity and mortality in HIV infected patients (Obaro, 2009). The prevalence of Streptococcus pneumoniae isolates used for this study in both HIV seronegative and HIV seropositive patients were $29(16.1 \%)$ and $24(20.0 \%)$ respectively. There was no significant difference with the prevalence of $S$ .pneumoniae among the HIV seropositive and seronegative patients $(P>0.05)$. Shailaja et al. (2004) however had a higher prevalence of $44.28 \%$ amongst 100 HIV seropositive patients. The prevalence value here is much higher considering the fact that they studied HIV seropositive patients who were not on any antibiotic prophylaxis and also worked on $30 \mathrm{HIV}$ seronegative patients as controls but failed to report the prevalence value in this category of patients.

The antibiotic susceptibility profile patterns for both HIV seropositive patients and HIV seronegative patients tested in this present study showed that Ciprofloxacin 25(86.2\%), was most active invitro followed by Oxacillin 21(72.4\%), Augmentin 19(65.5), Chloramphenicol 15(51.7\%), Tetracycline, 14(48.3\%), Clindamycin 14(48.3) and Erythromycin 10(34.5\%) for HIV seropositive patients respectively. While for HIV seronegative patients antibiotic susceptibility profile showed that Oxacillin 20(83.3\%), was most active followed by Augmentin 20(83.3\%), Ciprofloxacin 18(75.0\%), Clindamycin 15(62.5), Erythromycin $13(54.2 \%)$ Tetracycline $11(45.8 \%)$ in HIV seronegative patients, respectively. There was no significant difference in the levels of antibiotic susceptibilities and resistant observed $(p>$ 0.05 ). These imply that the HIV status of patients did not influence antimicrobial susceptibility of the Streptococcus pneumoniae isolates among the HIV/AIDS seropositive and HIV seronegative Patients. This also agreed with work described by Ndip et al. (1995) who had previously reported that sensitivity to penicillin and erythromycin by $S$. pneumoniae isolates in 1995. Also in hospital-based study by Akanbi et al. (2004) in 2002 in Ilorin, Nigeria, the recovered $S$. pneumoniae isolates were resistant to the tested antibiotics, including penicillin (83\%), erythromycin (56.6\%), ceftriaxone $(28 \%)$, ciprofloxacin $(20 \%)$ and ampicillin (73.8\%).

From the study is was found out that the $\mathrm{CD}_{4}$ cells counts for the HIV seropositive patients were $161(89.4 \%) 350$ cells $/ \mathrm{ml}$ and $19(10.6 \%)$ $<350$ cells $/ \mathrm{m}$ recorded as normal and low respectively. HIV-infected persons with low CD4 counts may be at highest risk for colonization and consequently for invasive disease. CD4 counts less than 350 have been associated with increased risk of invasive disease in some studies as reported by (Feikin et al,. 2004). In this study, t- test analysis was carried out to correlate the CD4 cells counts of the study population with the prevalence of Streptococcus pneumoniae isolates among the HIV seropositive patients. There was significant difference ( $p$ $<0.05$ ) for the CD4 cells count of the study population with the prevalence of Streptococcus pneumoniae isolates among the HIV seropositive patients. In Contrary to the publicized reports by Nicoletti et al.(2007)who found that neither the CD4 nor the level HIV of viral load was significantly associated with pneumococcal infection among the HIV patients.

In our study, only two (2) (33.3\%) out of six(6) resistant isolates were observed to posses penicillin resistant genes that detect the pneumococcal pbp2b. The pbp2b gene was not detected in four (4) resistant pneumococci in our study, the source of resistance to penicillin in these strains can be caused by mutations in other genes that code the residual PBPs.

Penicillin resistant streptococcus pneumoniae (PRSP) isolates containing the pbp2b gene were the most frequently found.. It was found by Habibian et al. (2013) that the pbp2b gene was found in 4 of the total 10 samples (Habibian et al., 2013). Also Kotevska et al., (2009) reported that of the total 40 resistant/ intermediate resistant S.pneumoniae, 22 genes pbp2b and/or pbp2x were verified. This is higher than $4 \%$ from Sweden (Skovbjerg et al., 2013), 7.2\% from Germany (Imohl et al., 2015), 7.8\% reported from India (Song et al., 2004), but less than 36.9\% from Spain ( Fenoll et al., 2000), $27.95 \%$ from South Korea (Song et al., 2015), $22.8 \%$ from France (Lee et al,. 2010), with the least being $94.5 \%$ reported from Hamadan, Iran (Cohen et al,. 2002). Previous researches have obviously pointed out that the three penicillinbinding proteins (PBP 1a, 2x and 2b) are important for the resistance to beta-lactams.

\section{CONCLUSION}

In this study, there was no significant difference in the prevalence of $S$.pneumoniae among the HIV seropositive and seronegative patients ( $P$ $>0.05)$. But the age group (30-39) years was recorded the highest prevalence of Streptococcus pneumoniae isolates in HIV seropositive patients and the age group (10-19) years recorded the highest prevalence of Streptococcus pneumoniae isolates in HIV serongative patients. 
Special Conference Edition, November, 2019

This age group are active and therefore exposed to various predisposing factor(s). Result also showed that Streptococcus pneumoniae isolates was susceptible to the antibiotics tested in order Ciprofloxacin $>$ Oxacillin $>$ Augmentin $>$ Chloramphenicol > Tetracycline $>$ Clindamycin and Erythromycin. On the other hand the isolates are resistant to antibiotics in order Erythromycin > Chloramphenicol, Tetracycline, $>$ Clindamycin, >Augmentin > Oxacillin > Ciprofloxacin. There was no significant difference in the levels of antibiotic susceptibilities and resistant observed $(p>$ 0.05 ). The $C_{4}$ cells counts for the HIV seropositve patients were $161(89.4 \%) 350$ cells $/ \mathrm{ml}$ and $19(10.6 \%)<350$ cells $/ \mathrm{m}$ recorded as normal and low respectively. There was significant difference $(p<0.05)$ for the CD4 cells counts of the study population with the prevalence of Streptococcus pneumoniae isolates among the HIV seropositive patients. PCR implication revealed out that Only two(2) $(33.3 \%)$ out of six(6) resistance isolates were observed penicillin resistance genes pneumococcal pbp2b genes

\section{REFERENCES}

Adeleye, A,. Uju, L,. Idika, N. and Sobande, O. (2008). Cotrimoxazole resistance in Streptococcus pneumoniae isolated from sputum of HIV-positive patients. West Indian medical Journal 57 no.5.

Akanbi, I.I., Taiwo, A.A., Babatunde, S.S., Onilke, S.K. and B.A, Abdulraheem, I.S. (2004). Antibiotic susceptibility pattern of Streptococcus pneumoniae in Ilorin, Nigeria. African. Journal of Clinical . Experimental Microbiolology. 5:173176.

Clinical and Laboratory Standards institute (CLSI) (2010). Methods for the dilution, antimicrobial susceptibility test for bacteria. Approved standards. Ninth Editions (M09).

Cohen, M. L. (2002). Epidemiology of drug resistance, implications for a postantimicrobial era. Science, 257: 10501055.

Cohen, A.L., Harrison, L.H, Farley, M.M, Reingold, A.L, Hadler, J. and Schaffner. W. 2010). Prevention of invasive pneumococcal disease among HIVinfected adults in the era of childhood pneumococcal immunization. AIDS Lond England;24:2253-62.

Feikin, D.R., Feldman., C., Schuchat, A. and Janoff, E.N. (2004). Global strategies to prevent bacterial pneumonia in adults with HIV disease. Lancet Infection of Disease.;4(7):445-55.

\section{RECOMMENDATIONS}

1. Most of the Streptococcus pneumoniae associated infection in AIDS clients, go undetected hence the importance of this study. Patients presenting with respiratory failure should undergo adequate analysis and examined before critical care for treatment and support to know exact causative agent especially in immune compromised individuals.

2. Antibiotic susceptibility testing should be done routinely in all S.pneumoniae clinical isolates from HIV patients, regardless of their source.

3. Patients with $<350$ CD4 cells are at a greater risk for pneumococcal disease and are less likely to have an adequate antibody response to pneumococcal immunization. Therefore pneumococcal vaccination should be given as early as possible after HIV infection is diagnosed.

4. In order to curb the problem of antibiotic resistance, indiscriminate use of antibiotics and over the counter sales of antibiotics should be discouraged and avoided.

Fenoll, A., Aguilar, L., Gimenez, M.J., Vicioso, M.D., Robledo, O. and Granizo J.J. ( 2012). Variations in serotypes and susceptibility of adult non-invasive Streptococcus pneumoniae isolates between the periods before (May 2000May 2001) and 10 years after (May 2010-May 2011) introduction of conjugate vaccines for child immunisation in Spain. International Journal Antimicrobial Agents.;40(1):1823.

Habibian, Mehrabi-Tavana , Ahmadi , Izadi, Jonaidi and Darakhshanpour (2013) . Serotype distribution and antibiotics susceptibility pattern of Streptococcus pneumonia in Iran. Iran Red Crescent Med J.;15(10):8053.

Huang, L.F., Tang, X.P., Cai, W.P., Chen, X.J., Lei, C.L., Li, L.H. and Zhang F.C.. (2010). An analysis of opportunistic infection in 762 patients with human immunodeficiency virus infection in Guangdong areas. Zhonghua Nei Ke Za Zhi 49(8):653-6.

Theodoratou, E., McAllister, D.A., Reed, C., Adeloye, D.O., Rudan, I. and Muhe, L.M.( 2014) . Global, regional, and national estimates of pneumonia burden in HIV-infected children in 2010: a meta-analysis and modeling study. Lancet Infections Diseasas, 14:1250-8. 
Special Conference Edition, November, 2019

Thornhill, J., Sivaramakrishnan, A. and Orkin, C. (2014.)Pneumococcal vaccination in people living with HIV. Vaccine

Imohl, M., Reinert, R.R. and Linden, M. (2015). Antibiotic susceptibility rates of invasive pneumococci before and after the introduction of pneumococcal conjugate vaccination in Germany. International Journal of Medical Microbiology:; 305(7):776-83.

Kotevska, V., Trajkovska-Dokic, E., Jankoska, G., Kaftandzieva, A., Panovski, N. and Petrovska M. (2009). Phenotypes and genes of resistance of pneumococci to penicillin isolated from children. ;30(1):143-54.

Lee, H.J., Park, J.Y and Jang, S.H. (1995). High incidence of resistance to multiple antimicrobials in clinical isolates of Streptococcus pneumoniae from a university hospital in Korea. Clin Infect Dis. ; 20:826-35.

Ndip, R.N., Obi, M.C., Obi, C.L, Nwawolo, C., Igumbor, E.O. and Obi, A.A. (1995). Antibiogram of bacterial isolates from cases of otitis media and lower respiratory tract infections. African Journal of Medical Microbiology of Science. 24:353-357.

Nicoletti, C., M. C. Brandileone, M. L. Guerra, and A. S. Levin. (2007). Prevalence, serotypes, and risk factors for pneumococcal carriage among HIVinfected adults. Diagnostic .Microbiology and Infection of Diseases . 57:259265.

Ochei, J. and Kolkhtar, A. (2008). Bacteriology: Medical Laboratory Science, theory and practice. In:Bulakh P.M. and Deshmukh S. (eds). Tata McGraw-Hill publishing
Company limited New Delhi. Pp. 525752.

Obaro, S. (2009). Seven-valent pneumococcal conjugate vaccines for developing countries. Expert Review of Vaccines. 8:1051-1061.

Oluyege, A.O., Dada, A.C., Ojo, A.M. and Oluwadare, E (2009). Antibiotics resistance profile of bacterial isolates from food sold on University campuses in South West, Nigeria. Journal of Biotechnology. 8 (21) 5883-5887.

Rano, A., Agusti, C., Sibila, O. and Torres, A (2005). Pulmonary infections in nonHIV-immunocompromised patients. Curr Opin Pulmonary Medical 11 (3): 213217. WHO (2008). "Causes of death in neonates and children under five in the world 2004".

Song, J.H., Jung, S.I., Ko, K.S, Kim, N.Y., Son, J.S and Chang, HH. (2004). High prevalence of antimicrobial resistance among clinical Streptococcus pneumoniae isolates in Asia (an ANSORP study). Antimicrobial Agents Chemotheraphy.;48(6):2101-7.

Sedigheh, R. T., Mohammad,R., , Ali, N. A., Fatemeh, F. and Ali Hashemi. (2017), Detection of $p b p 2 b$ Gene and Antimicrobial Susceptibility Pattern of Streptococcus Pneumoniae Isolates in Tehran Hospitals. Arch PediatrInfect Dis. 5(1):e38891.

Shailaja, V.V., Pai, L.A., Mathur, D.R,. and Lakshmi, V (2004). Prevalence of Bacterial and Fungal Agents Causing Lower Respiratory Tract Infections in Patients with Human Immunodeficiency Virus Infection. Indian Journal of Medical Microbiology, 22 (1):28-33. 